\title{
Direct Quantification of Inner and Outer-sphere Oxyanion Complexation Kinetics Using Spectroscopic Analysis: Ionic Strength and Background Cation Effect on Sulfate Adsorption to Hematite
} AND DEREK PEAK ${ }^{1}$

${ }^{1}$ Department of Soil Science, College of Agriculture and Bioresources, University of Saskatchewan, Saskatoon, SK S7N 5A8, Canada Email: (mps286@usask.ca)

Sulfate adsorbs on Fe-oxide minerals by inner- and outersphere modes, however, the time dependence of coexisting surface species is not clear. Using in situ attenuated total reflectance-Fourier transform infrared spectroscopy (ATRFTIR) and multivariate curve resolution (MCR) analyses, we quantify adsorption and desorption kinetics of inner- and outer-sphere sulfate species on hematite at two ionic strengths $(\mathrm{I}=0.01$ and $0.10 \mathrm{M})$ and background cations $(\mathrm{K}+$ and $\mathrm{Ca}+2)$ at $\mathrm{pH}$ 4.5. We experimentally observed inner-sphere, bidentate bridging and outer-sphere species kinetics congruent with the proposed stepwise Eigen-Werner-Wilkins mechanism, in which a rapid formation of outer-sphere association precedes a slower conversion of outer-sphere to inner-sphere sulfate complexes. The rate limitation imposed by inner-sphere complex formation is likely linked with displacement of protonated surface hydroxyl groups on the oxide surface by the adsorbing oxyanion. Outer-sphere complexes are responsible for rapid adsorption and desorption seen in total sulfate, whereas inner-sphere species desorb more slowly and partially reflect sulfate recalcitrant to desorption. This work presents a novel, direct spectroscopic quantification of mixed surface species adsorption kinetics on a model mineral surface which may be used to confirm proposed molecular mechanisms for other oxyanion adsorption to mineral surfaces. Our results enhance molecular-level understanding of oxyanion adsorption on soils and help predict their behavior in soils. 